\title{
On the quasilinear elliptic problem with a Hardy-Sobolev critical exponent
}

\author{
Guanwei Chen and Shiwang Ma
}

Communicated by Shiyi Chen, received April 9, 2010.

\begin{abstract}
In this article, we consider a quasilinear elliptic equation involving Hardy-Sobolev critical exponents and superlinear nonlinearity. The right hand side nonlinearity $f(x, u)$ which is $(p-1)$-superlinear nearby 0 . However, it does not satisfy the usual Ambrosetti-Rabinowitz condition (AR-condition). Instead we employ a more general condition. Using a variational approach based on the critical point theory and the Ekeland variational principle, we show the existence of two nontrivial positive solutions. Moreover, the obtained results extend some existing ones.
\end{abstract}

\section{Contents}

1. Introduction and main results

2. Proof of Theorem 1

3. Proof of Theorem $2 \quad 229$

Appendix A 236

References 236

\section{Introduction and main results}

We will consider the following problem

$$
\left\{\begin{array}{l}
-\triangle_{p} u=\mu \frac{|u|^{p^{*}(s)-2}}{|x|^{s}} u+\lambda f(x, u), x \in \Omega \backslash\{0\} \\
u=0, x \in \partial \Omega
\end{array}\right.
$$

where $\triangle_{p} u=\operatorname{div}\left(|\nabla u|^{p-2} \nabla u\right)$ denotes the $p$-laplacian differential operator, $\Omega$ is an open bounded domain in $R^{N}(N \geq 3)$ with smooth boundary $\partial \Omega$ and $0 \in \Omega, 0 \leq$

1991 Mathematics Subject Classification. 35A15, 35K91.

Key words and phrases. p-Laplacian; Hardy-Sobolev critical exponent; $(\mathrm{PS})_{c^{-c o n d i t i o n}}$ Mountain pass lemma; Ekeland variational principle.

Research supported by the Specialized Fund for the Doctoral Program of Higher Education and the National Natural Science Foundation of China. 
$s<p, 1<p<N, 0<\mu<\infty, p^{*}(s)=\frac{p(N-s)}{N-p}$ is the Hardy-Sobolev critical exponent and $p^{*}=p^{*}(0)=\frac{N p}{N-p}$ is the Sobolev critical exponent, $\lambda>0$ is a real parameter. $W_{0}^{1, p}(\Omega)$ is the Sobolev space with the norm

$$
\|u\|:=\left(\int_{\Omega}|\nabla u|^{p} d x\right)^{\frac{1}{p}}
$$

which is equivalent to the usual norm of $W_{0}^{1, p}(\Omega)$ due to the Poincaré inequality and

$$
A_{s}(\Omega):=\inf _{u \in W_{0}^{1, p}(\Omega) \backslash\{0\}} \frac{\|u\|^{p}}{\left(\int_{\Omega} \frac{|u|^{p^{*}(s)}}{|x|^{s}} d x\right)^{\frac{p}{p^{*}(s)}}}
$$

is the best Hardy-Sobolev constant.

In the case $s=0$ and $p=2$, this problem has been widely studied (see $[\mathbf{1}, \mathbf{2}, \mathbf{1 1}]$ and the references therein). In the case $s=0$, Goncalves and Alves in [8] have studied Problem (1.1) in $R^{N}$ involving $f(x, u)=h(x) u^{q}, u \geq 0$ and $u \not \equiv 0$ to obtain existence of positive solutions where $2 \leq p<N, 0<q<p-1$ or $p-1<q<p^{*}-1$ and a suitable $h$. Ghoussoub and Yuan have studied problem (1.1)(see [9]), when $f(x, u)=|u|^{r-2} u, p \leq r \leq p^{*}$. For other relevant papers see $[\mathbf{6}, \mathbf{1 0}, \mathbf{7}, \mathbf{1 2}]$ and the references herein.

A direct extension of these methods to the case $p \neq 2$ is faced with serious difficulties. Such as, the energy functional associated to (1.1) is defined on $W_{0}^{1, p}(\Omega)$, which is not a Hilbert space for $p \neq 2$. Due to the lack of compactness of the embedding in $W_{0}^{1, p}(\Omega) \hookrightarrow L^{p^{*}}(\Omega)$ and $W_{0}^{1, p}(\Omega) \hookrightarrow L^{p^{*}(s)}\left(\Omega,|x|^{-s} d x\right)$, we cannot use the standard variational argument directly. The corresponding energy functional fails to satisfy the classical Palais-Smale ((PS) for short) condition in $W_{0}^{1, p}(\Omega)$. However, a local (PS) condition can be established in a suitable range. Then the existence result is obtained via constructing a minimax level within this range and the Mountain Pass Lemma due to A. Ambrosetti and P.H. Rabinowitz (see also $[15])$.

$F(x, t)$ is a primitive function of $f(x, t)$ defined by $F(x, t):=\int_{0}^{t} f(x, s) d s$ for $x \in \Omega, t \in R$. For problem (1.1) we have the following assumptions:

$$
\left(A_{1}\right) f \in C\left(\bar{\Omega} \times R^{+}, R\right), f(x, 0) \equiv 0, \lim _{t \rightarrow 0^{+}} \frac{f(x, t)}{t^{p-1}}=+\infty \text { and } \lim _{t \rightarrow \infty} \frac{f(x, t)}{t^{p^{*}}(s)-1}=0
$$

uniformly for $x \in \bar{\Omega}$.

$\left(A_{2}\right) f: \Omega \times R^{+} \rightarrow R$ is nondecreasing with respect to the second variable.

$$
\left(A_{3}\right) N>p \geq \max \left\{2, \frac{3 N}{N+3-s}, \frac{s-1+\sqrt{(1-s)^{2}+4 N}}{2}\right\} \text {. }
$$

In what follows, $\|\cdot\|_{p}$ denotes the norm in $L^{p}(\Omega)$. Now, our main results are as follows:

Theorem 1. Suppose that $N \geq 3,0<\mu<\infty, 0 \leq s<p, 1<p<N$. Assume $\left(A_{1}\right)$ holds, then there exists $\lambda^{*}>0$ such that problem (1.1) has at least one nontrivial positive solution $u_{\lambda}$ for every $\lambda \in\left(0, \lambda^{*}\right)$.

Theorem 2. Suppose that $N \geq 3,0<\mu<\infty, \max \left\{0, \frac{p^{2}-N}{p-1}\right\}<s<p$. If $\left(A_{1}\right)-\left(A_{3}\right)$ all hold, then there exists $\lambda^{*}>0$ such that problem (1.1) has at least two nontrivial positive solutions for every $\lambda \in\left(0, \lambda^{*}\right)$. 
Remark 1. Here we give some examples of the nonlinearity satisfying $\left(A_{1}\right)$ and $\left(A_{2}\right)$.

(1) $f(x, t)=t^{q-1}, t \geq 0$ with $1<q<p$.

(2) $f(x, t)=v(x) t^{r}+h(x) t^{\nu}, t \geq 0$, where $v(x), h(x) \in L^{\infty}(\Omega), v(x), h(x)>$ $0,0 \leq r<p-1$ and $p-1<\nu<p^{*}(s)-1$.

This paper is organized as follows. In Section 2, we manage to give the proof of Theorem 1. The proof of Theorem 2 is given in Section 3. Throughout the article the letters $C$ or $C_{i}(i=1,2,3, \ldots)$ will denote various positive constants whose exact value may change from line to line but are not essential to the analysis of the problem.

\section{Proof of Theorem 1}

It is obvious that the values of $f(x, t)$ for $t<0$ are irrelevant in Theorem $1-2$, and we may define

$$
f(x, t) \equiv 0 \text { for } x \in \Omega, t \leq 0 .
$$

Let $u^{ \pm}:=\max \{ \pm u, 0\}$. The functional corresponding to (1.1) is

$$
I(u)=\frac{1}{p} \int_{\Omega}|\nabla u|^{p} d x-\frac{\mu}{p^{*}(s)} \int_{\Omega} \frac{\left(u^{+}\right)^{p^{*}(s)}}{|x|^{s}} d x-\lambda \int_{\Omega} F\left(x, u^{+}\right) d x, u \in W_{0}^{1, p}(\Omega) .
$$

By Hardy-Sobolev inequalities (see $[4,9])$ and $\left(A_{1}\right), I \in C^{1}\left(W_{0}^{1, p}(\Omega), R\right)$. Now it is well known that there exists a one-to-one correspondence between the weak solutions of problem (1.1) and the critical points of $I$ on $W_{0}^{1, p}(\Omega)$. More precisely we say that $u \in W_{0}^{1, p}(\Omega)$ is a weak solution of problem (1.1), if for any $v \in W_{0}^{1, p}(\Omega)$, there holds

$$
\left\langle I^{\prime}(u), v\right\rangle=\int_{\Omega}|\nabla u|^{p-2} \nabla u \nabla v d x-\mu \int_{\Omega} \frac{\left(u^{+}\right)^{p^{*}(s)-1}}{|x|^{s}} v d x-\lambda \int_{\Omega} f\left(x, u^{+}\right) v d x=0 .
$$

Proof of Theorem 1. Let $X:=W_{0}^{1, p}(\Omega)$. From the Sobolev and Hardy-Sobolev inequalities, we can easily get

$$
\|u\|_{p}^{p} \leq C\|u\|^{p} ; \int_{\Omega} \frac{|u|^{p^{*}(s)}}{|x|^{s}} d x \leq C\|u\|^{p^{*}(s)} ;\|u\|_{p^{*}}^{p^{*}} \leq C\|u\|^{p^{*}}, \forall u \in X .
$$

It follows from $\left(A_{1}\right)$ that

$$
\begin{gathered}
\exists \delta>0 \text { such that }|F(x, t)|<\frac{t^{p^{*}(s)}}{p^{*}(s)\left|x^{s}\right|} \text { for } t>\delta, \\
\exists M_{1}>0 \text { such that }|F(x, t)| \leq M_{1} \text { for all } t \in[0, \delta],
\end{gathered}
$$

uniformly for all $x \in \bar{\Omega} \backslash\{0\}$. Therefore, we deduce that

$$
|F(x, t)| \leq M_{1}+\frac{t^{p^{*}(s)}}{p^{*}(s)\left|x^{s}\right|}
$$

for all $t \in R$ and for $x \in \bar{\Omega} \backslash\{0\}$. By (2.1) and (2.2), we have

$$
\begin{aligned}
& I(u)=\frac{1}{p}\|u\|^{p}-\frac{\mu}{p^{*}(s)} \int_{\Omega} \frac{\left(u^{+}\right)^{p^{*}(s)}}{|x|^{s}} d x-\lambda \int_{\Omega} F\left(x, u^{+}\right) d x \\
& \geq \frac{1}{p}\|u\|^{p}-C_{1}\|u\|^{p^{*}(s)}-\lambda M_{1}|\Omega|
\end{aligned}
$$


for all $\lambda \in(0,1]$ and some $C_{1}=\frac{C \mu}{p^{*}(s)}$, so there exist $\rho>0$ and $\lambda^{*} \in(0,1]$ such that

$$
I(u)>0 \text { if }\|u\|=\rho, \text { and } I(u) \geq-C_{2} \text { if }\|u\| \leq \rho
$$

for every $0<\lambda<\lambda^{*}$, where $C_{2}=C_{1} \rho^{p^{*}(s)}+\lambda^{*} M_{1}|\Omega|$. Choose $u_{0} \in W_{0}^{1, p}(\Omega) \cap$ $L^{\infty}(\Omega)$ such that $u_{0}^{+} \neq 0$. Let $M_{2}:=\left\|u_{0}\right\|^{p} /\left(\lambda\left\|u_{0}^{+}\right\|_{p}^{p}\right)$. From $\left(A_{1}\right)$, there exists $\delta_{1}$ such that

$$
|F(x, t)| \geq \frac{2 M_{2}}{p}|t|^{p}, 0<t<\delta_{1} .
$$

Hence we have

$$
\begin{aligned}
I\left(r u_{0}\right) & =\frac{r^{p}}{p}\left\|u_{0}\right\|^{p}-\frac{\mu r^{p^{*}(s)}}{p^{*}(s)} \int_{\Omega} \frac{\left(u_{0}^{+} p^{p^{*}(s)}\right.}{|x|^{s}} d x-\lambda \int_{\Omega} F\left(x, r u_{0}^{+}\right) d x \\
& \leq \frac{r^{p}}{p}\left\|u_{0}\right\|^{p}-\frac{2 r^{p}}{p} \lambda M_{2}\left\|u_{0}^{+}\right\|_{p}^{p} \\
& =-\frac{r^{p}}{p}\left\|u_{0}\right\|^{p}<0
\end{aligned}
$$

for every $0<\lambda<\lambda^{*}$ and $0<r<\min \left\{\rho, \delta_{1} /\left\|u_{0}^{+}\right\|_{\infty}\right\}$. Thus there exists $u$ small enough such that $I(u)<0$. Then we deduce that

$$
\inf _{u \in \overline{B_{\rho}(0)}} I(u)<0<\inf _{u \in \partial \overline{B_{\rho}(0)}} I(u) .
$$

By applying Ekeland's variational principle (see [13], Theorem 4.1) in $\overline{B_{\rho}(0)}$, there is a minimizing sequence $\left\{u_{n}\right\} \subset \overline{B_{\rho}(0)}$ such that

$$
I\left(u_{n}\right) \leq \inf _{u \in \overline{B_{\rho}(0)}} I(u)+\frac{1}{n}, \quad I(\omega) \geq I\left(u_{n}\right)-\frac{1}{n}\left\|\omega-u_{n}\right\|, \omega \in \overline{B_{\rho}(0)} .
$$

Therefore, we have

$$
\left\|I^{\prime}\left(u_{n}\right)\right\| \rightarrow 0 \text { and } I\left(u_{n}\right) \rightarrow c_{\lambda} \text { as } n \rightarrow \infty,
$$

where $c_{\lambda}$ stands for the infimum of $I(u)$ on $\overline{B_{\rho}(0)}$. Since $\left\{u_{n}\right\}$ is bounded and $\overline{B_{\rho}(0)}$ is a closed convex set, there exist $u_{\lambda} \in \overline{B_{\rho}(0)} \subset W_{0}^{1, p}(\Omega)$. Going if necessary to a subsequence, one can get that(see $[\mathbf{9}]$ )

$$
\left\{\begin{array}{l}
u_{n} \rightarrow u_{\lambda} \text { weakly in } W_{0}^{1, p}(\Omega), \\
u_{n} \rightarrow u_{\lambda} \text { strongly in } L^{\gamma}(\Omega), 1<\gamma<p^{*}, \\
u_{n} \rightarrow u_{\lambda} \text { a.e. in } \Omega \\
\nabla u_{n} \rightarrow \nabla u_{\lambda} \text { a.e. in } \Omega, \\
\frac{u_{n}}{x} \rightarrow \frac{u_{\lambda}}{x} \text { weakly in } L^{p}(\Omega), \\
\int_{\Omega} \frac{\left|u_{n}\right|^{p^{(}(s)-2} u_{n}}{|x|^{s}} v d x \rightarrow \int_{\Omega} \frac{\left|u_{\lambda}\right|^{p^{*}(s)-2} u_{\lambda}}{|x|^{s}} v d x, \forall v \in W_{0}^{1, p}(\Omega) .
\end{array}\right.
$$

Consequently, passing to the limit in $\left\langle I^{\prime}\left(u_{n}\right), v\right\rangle$, as $n \rightarrow \infty$, we have

$$
\int_{\Omega}\left|\nabla u_{\lambda}\right|^{p-2} \nabla u_{\lambda} \nabla v d x-\mu \int_{\Omega} \frac{\left(u_{\lambda}^{+}\right)^{p^{*}(s)-1} v}{|x|^{s}} d x-\lambda \int_{\Omega} f\left(x, u_{\lambda}^{+}\right) v d x=0
$$

for all $v \in W_{0}^{1, p}(\Omega)$. That is, $\left\langle I^{\prime}\left(u_{\lambda}\right), v\right\rangle=0$. Thus $u_{\lambda}$ is a critical point of the functional $I$. Since $\left\|u_{\lambda}^{-}\right\|^{p}=-\left\langle I^{\prime}\left(u_{\lambda}\right), u_{\lambda}^{-}\right\rangle=0$, thus $u_{\lambda}=u_{\lambda}^{+} \geq 0$. Moreover, we deduce from $\left(A_{1}\right)$ and the boundedness of $\Omega$ that

$$
\begin{aligned}
& \exists M_{3}>0 \text { such that }|f(x, t)|<\frac{\mu}{\lambda} \frac{p^{p^{*}(s)-1}}{|x|^{s}} \text { for } t>M_{3}, \\
& \exists \delta_{2} \in\left(0, M_{3}\right) \text { such that }|f(x, t)|>0 \text { for } 0<t<\delta_{2},
\end{aligned}
$$


$\exists M_{4}>0$ such that $|f(x, t)| \leq M_{4}$ for all $t \in\left[\delta_{2}, M_{3}\right]$,

for all $x \in \bar{\Omega} \backslash\{0\}$. Therefore, we deduce that

$$
f(x, t) \geq-\frac{\mu}{\lambda} \frac{t^{p^{*}(s)-1}}{|x|^{s}}-M_{4} t \delta_{2}^{-1}
$$

for all $t \in R^{+}$and for $x \in \bar{\Omega} \backslash\{0\}$. From (1.1) and (2.3), we have

$$
-\triangle_{p} u_{\lambda}+\lambda M_{4} \delta_{2}^{-1} u_{\lambda} \geq 0 \text {. }
$$

From the strong maximum principle, we deduce that $u_{\lambda}>0$. So Theorem 1 is proved.

\section{Proof of Theorem 2}

The first positive solution $u_{\lambda}$ of problem (1.1) have been obtained in previous section, we can look for the second positive solution by a translated functional as in [1]. For fixed $\lambda \in\left(0, \lambda^{*}\right)$, we look for the second solution of problem (1.1) of the form $u=u_{\lambda}+v$, where $u_{\lambda}$ is the first positive solution obtained in previous section. The corresponding equation for $v$ is

$$
\left\{\begin{array}{l}
-\triangle_{p} v=\mu \frac{\left(u_{\lambda}+v\right)^{p^{*}(s)-1}}{|x|^{s}}-\mu \frac{\left(u_{\lambda}\right)^{p^{*}(s)-1}}{|x|^{s}}+\lambda f\left(x, u_{\lambda}+v\right)-\lambda f\left(x, u_{\lambda}\right), x \in \Omega \backslash\{0\} \\
v=0, x \in \partial \Omega .
\end{array}\right.
$$

Let us define

$$
\begin{gathered}
g(x, t)=\left\{\begin{array}{l}
\mu \frac{\left(u_{\lambda}+t\right)^{p^{*}(s)-1}}{|x|^{s}}-\mu \frac{\left(u_{\lambda}\right)^{p^{*}(s)-1}}{|x|^{s}}+\lambda f\left(x, u_{\lambda}+t\right)-\lambda f\left(x, u_{\lambda}\right), t \geq 0, \\
0, t<0,
\end{array}\right. \\
G(x, t)=\int_{0}^{t} g(x, s) d s
\end{gathered}
$$

and

$$
\begin{aligned}
J(v)= & \frac{1}{p} \int_{\Omega}|\nabla v|^{p} d x-\int_{\Omega} G\left(x, v^{+}\right) d x \\
= & \frac{1}{p}\|v\|^{p}-\frac{\mu}{p^{*}(s)} \int_{\Omega}\left(\frac{\left(u_{\lambda}+v^{+}\right)^{p^{*}(s)}}{|x|^{s}}-\frac{u^{p^{*}(s)}}{|x|^{s}}-p^{*}(s) \frac{u_{\lambda}^{p^{*}(s)-1} v^{+}}{|x|^{s}}\right) d x \\
& -\lambda \int_{\Omega}\left[F\left(x, u_{\lambda}+v^{+}\right)-F\left(x, u_{\lambda}\right)-f\left(x, u_{\lambda}\right) v^{+}\right] d x .
\end{aligned}
$$

Now we have one-to-one correspondence between critical points of $J$ in $W_{0}^{1, p}(\Omega)$ and solutions of problem (3.1). That is, if $v \in W_{0}^{1, p}(\Omega), v \neq 0$ is a critical point of $J$, then $v$ is a solution of (3.1). Since $\left\|v^{-}\right\|^{p}=-\left\langle J^{\prime}(v), v^{-}\right\rangle=0$, thus $v=v^{+} \geq 0$. Moreover, by the Maximum Principle, $v>0$ in $\Omega$. Here $u=u_{\lambda}+v$ is a positive solution of (1.1) and $u \neq u_{\lambda}$. We will prove the existence of a second positive solution of (1.1) by contradiction. Assume that $v=0$ is the only critical point of $J$ in $W_{0}^{1, p}(\Omega)$.

Lemma 1. $v=0$ is a local minimum of $J$ in $W_{0}^{1, p}(\Omega)$.

Proof. For any $v \in W_{0}^{1, p}(\Omega)$, write $v=v^{+}-v^{-}$. From the expression of $J$ and direct computation, we obtain that

$$
J(v)=\frac{1}{p}\left\|v^{-}\right\|^{p}+I\left(u_{\lambda}+v^{+}\right)-I\left(u_{\lambda}\right) .
$$


Since $u_{\lambda}$ is a local minimizer of $I$ in $W_{0}^{1, p}(\Omega)$, we have

$$
J(v) \geq \frac{1}{p}\left\|v^{-}\right\|^{p}
$$

as long as $\|v\| \leq \varepsilon$ for $\varepsilon$ small enough.

Lemma 2. ([9]) Suppose $1<p<N, 0 \leq s<p$. Then we have the following:

(i) $A_{s}(\Omega)$ is independent of $\Omega$ (and will henceforth be denoted by $A_{s}$ ).

(ii) $A_{s}$ is attained when $\Omega=\mathrm{R}^{N}$ by the functions

$$
l_{\varepsilon}(x)=\left[\varepsilon(N-s)\left(\frac{N-p}{p-1}\right)^{p-1}\right]^{\frac{N-p}{p(p-s)}}\left(\varepsilon+|x|^{\frac{p-s}{p-1}}\right)^{\frac{p-N}{p-s}}
$$

for some $\varepsilon>0$. Moreover the functions $l_{\varepsilon}(x)$ are the only positive radial solutions of

in $\mathrm{R}^{N}$, and satisfy

$$
-\triangle_{p} u=\frac{u^{p^{*}(s)-1}}{|x|^{s}}
$$

$$
\int_{R^{N}}\left|\nabla l_{\varepsilon}\right|^{p} d x=\int_{R^{N}} \frac{\left|l_{\varepsilon}\right|^{p^{*}(s)}}{|x|^{s}} d x=A_{s}^{\frac{N-s}{p-s}} .
$$

Lemma 3. Suppose $0<\mu<\infty$, $f$ satisfies $\left(A_{1}\right)-\left(A_{3}\right)$. Assume that $v=0$ is the only critical point of J. Let $\left\{v_{n}\right\}$ be a $(P S)_{c}$ sequence with $0<c<\frac{p-s}{p(N-s)} A_{s}^{\frac{N-s}{p-s}} \mu^{\frac{p-N}{p-s}}$. Then we have

$$
v_{n} \rightarrow 0 \text { in } W_{0}^{1, p}(\Omega) \text { as } n \rightarrow \infty .
$$

Proof. Let $v_{n}$ be a sequence in $W_{0}^{1, p}(\Omega)$ such that

$$
J\left(v_{n}\right) \rightarrow c<\frac{p-s}{p(N-s)} A_{s}^{\frac{N-s}{p-s}} \mu^{\frac{p-N}{p-s}} \text { and } J^{\prime}\left(v_{n}\right) \rightarrow 0 \text { in }\left(W_{0}^{1, p}(\Omega)\right)^{*} .
$$

Then from (3.3) and (3.4), we have

$$
\begin{gathered}
J\left(v_{n}\right)=\frac{1}{p}\left\|v_{n}^{-}\right\|^{p}+I\left(u_{\lambda}+v_{n}^{+}\right)-I\left(u_{\lambda}\right)=c+o(1) \\
\left\langle J^{\prime}\left(v_{n}\right), u_{\lambda}+v_{n}^{+}\right\rangle=\int_{\Omega}\left|\nabla v_{n}^{-}\right|^{p-2} \nabla v_{n}^{-} \nabla u_{\lambda} d x+\left\langle I^{\prime}\left(u_{\lambda}+v_{n}^{+}\right), u_{\lambda}+v_{n}^{+}\right\rangle=o(1)\left\|u_{\lambda}+v_{n}^{+}\right\| .
\end{gathered}
$$

It yields that

$$
\begin{aligned}
& J\left(v_{n}\right)-\frac{1}{p}\left\langle J^{\prime}\left(v_{n}\right), u_{\lambda}+v_{n}^{+}\right\rangle \\
& =\frac{1}{p}\left(\left\|v_{n}^{-}\right\|^{p}-\int_{\Omega}\left|\nabla v_{n}^{-}\right|^{p-2} \nabla v_{n}^{-} \nabla u_{\lambda} d x-\left\langle I^{\prime}\left(u_{\lambda}+v_{n}^{+}\right), u_{\lambda}+v_{n}^{+}\right\rangle\right) \\
& +I\left(u_{\lambda}+v_{n}^{+}\right)-I\left(u_{\lambda}\right) \\
& \leq c+1+o(1)\left\|u_{\lambda}+v_{n}^{+}\right\| .
\end{aligned}
$$

Therefore, one gets

$$
\begin{aligned}
& \frac{1}{p}\left(\left\|v_{n}^{-}\right\|^{p}-\int_{\Omega}\left|\nabla v_{n}^{-}\right|^{p-2} \nabla v_{n}^{-} \nabla u_{\lambda} d x\right)+\mu\left(\frac{1}{p}-\frac{1}{p^{*}(s)}\right) \int_{\Omega} \frac{\left(u_{\lambda}+v_{n}^{+}\right)^{p^{*}(s)}}{|x|^{s}} d x \\
& +\lambda \int_{\Omega}\left[\frac{1}{p} f\left(x, u_{\lambda}+v_{n}^{+}\right)\left(u_{\lambda}+v_{n}^{+}\right)-F\left(x, u_{\lambda}+v_{n}^{+}\right)\right] d x \\
& \leq I\left(u_{\lambda}\right)+c+1+o(1)\left\|u_{\lambda}+v_{n}^{+}\right\| .
\end{aligned}
$$


By $\left(A_{1}\right)$ and the boundedness of $\Omega$, for any $\varepsilon>0$, there exists $M_{5}=M_{5}(\varepsilon)>0$ such that

$|f(x, t) t| \leq \varepsilon \frac{|t|^{p^{*}(s)}}{|x|^{s}}, x \in \Omega \backslash\{0\},|t|>M_{5}, \quad|f(x, t) t| \leq C_{3}(\varepsilon), x \in \Omega,|t| \in\left[0, M_{5}\right]$,

$|F(x, t)| \leq \frac{\varepsilon}{p} \frac{|t|^{p^{*}(s)}}{|x|^{s}}, x \in \Omega \backslash\{0\},|t|>M_{5}, \quad|F(x, t)| \leq C_{4}(\varepsilon), x \in \Omega,|t| \in\left[0, M_{5}\right]$, where $C_{3}(\varepsilon), C_{4}(\varepsilon)>0$. Therefore, we have

$$
\begin{aligned}
& |f(x, t) t| \leq C_{3}(\varepsilon)+\varepsilon \frac{|t|^{p^{*}(s)}}{|x|^{s}},(x, t) \in(\Omega \backslash\{0\}) \times R, \\
& |F(x, t)| \leq C_{4}(\varepsilon)+\frac{\varepsilon}{p} \frac{|t|^{p^{*}(s)}}{|x|^{s}},(x, t) \in(\Omega \backslash\{0\}) \times R .
\end{aligned}
$$

Let $C(\varepsilon):=\frac{1}{p} C_{3}(\varepsilon)+C_{4}(\varepsilon)$, combining (3.7) and (3.8), one gets

$$
F(x, t)-\frac{1}{p} f(x, t) t \leq C(\varepsilon)+\frac{2 \varepsilon}{p} \frac{|t|^{p^{*}(s)}}{|x|^{s}},(x, t) \in(\Omega \backslash\{0\}) \times R .
$$

From (3.6) and (3.9), we deduce that

$$
\begin{aligned}
& \left(\frac{\mu(p-s)}{p(N-s)}-\frac{2 \lambda \varepsilon}{p}\right) \int_{\Omega} \frac{\left(u_{\lambda}+v_{n}^{+}\right)^{p^{*}(s)}}{|x|^{s}} d x \\
& \leq \lambda C(\varepsilon)|\Omega|-\frac{1}{p}\left\|v_{n}^{-}\right\|^{p}+C_{5}\left\|v_{n}^{-}\right\|^{p-1}+C_{6}+o(1)\left\|u_{\lambda}+v_{n}^{+}\right\|,
\end{aligned}
$$

where $C_{5}=\frac{1}{p}\left\|u_{\lambda}\right\|, C_{6}=I\left(u_{\lambda}\right)+c+1$. Let $\varepsilon=\frac{\mu(p-s)}{4(N-s) \lambda}$, we have

$$
\int_{\Omega} \frac{\left(u_{\lambda}+v_{n}^{+}\right)^{p^{*}(s)}}{|x|^{s}} d x \leq C_{7}\left\|v_{n}^{-}\right\|^{p-1}+C_{8}+o(1)\left\|u_{\lambda}+v_{n}^{+}\right\|,
$$

where $C_{7}=\frac{2 p(N-s)}{\mu(p-s)} C_{5}, C_{8}=\frac{2 p(N-s)}{\mu(p-s)}\left(\lambda C(\varepsilon)|\Omega|+C_{6}\right)$, which together with (3.3), (3.5) and (3.8) imply that

$$
\begin{aligned}
& \frac{1-\varepsilon}{p}\left\|v_{n}^{-}\right\|^{p}+\frac{1}{p}\left[(1-\varepsilon)\left\|v_{n}^{+}\right\|^{p}-\overline{C_{\varepsilon}}\left\|u_{\lambda}\right\|^{p}-(1-\varepsilon)\left\|v_{n}^{+}\right\|^{p-1}\right] \\
& \leq \frac{1}{p}\left\|v_{n}^{-}\right\|^{p}+\frac{1}{p}\left[(1-\varepsilon)\left\|v_{n}^{+}\right\|^{p}-\overline{C_{\varepsilon}}\left\|u_{\lambda}\right\|^{p}\right] \\
& \leq \frac{1}{p}\left\|v_{n}^{-}\right\|^{p}+\frac{1}{p}\left|\left(\left\|v_{n}^{+}\right\|-\left\|u_{\lambda}\right\|\right)\right|^{p} \\
& \leq \frac{1}{p}\left\|v_{n}^{-}\right\|^{p}+\frac{1}{p}\left\|u_{\lambda}+v_{n}^{+}\right\|^{p} \\
& =\frac{\mu}{p^{*}(s)} \int_{\Omega} \frac{\left(u_{\lambda}+v_{n}^{+}\right)^{p^{*}(s)}}{|x|^{s}} d x+\lambda \int_{\Omega} F\left(x, u_{\lambda}+v_{n}^{+}\right) d x+J\left(v_{n}\right)+I\left(u_{\lambda}\right)+o(1) \\
& \leq C_{9}\left\|v_{n}^{-}\right\|^{p-1}+C_{10}+o(1)\left\|u_{\lambda}+v_{n}^{+}\right\|,
\end{aligned}
$$

where in the second step we used the fact that, the elementary inequality $|a-b|^{t} \geq$ $(1-\varepsilon) a^{t}-\overline{C_{\varepsilon}} b^{t}(t \geq 1, a, b>0)$ holds. $C_{9}=\left(\frac{\mu}{p^{*}(s)}+\frac{\lambda \varepsilon}{p}\right) C_{7}, C_{10}=\lambda C_{4}(\varepsilon)|\Omega|+$ $\left(\frac{\mu}{p^{*}(s)}+\frac{\lambda \varepsilon}{p}\right) C_{8}+I\left(u_{\lambda}\right)+c+o(1)$. Since $\left\|v_{n}^{-}\right\|^{p-1}+\left\|v_{n}^{+}\right\|^{p-1}=\left\|v_{n}\right\|^{p-1}$, then we deduce

$$
\left\|v_{n}\right\|^{p}-C_{11}\left\|v_{n}^{+}\right\|^{p-1}-C_{11}^{\prime}\left\|v_{n}^{-}\right\|^{p-1} \leq C_{12}+o(1)\left\|u_{\lambda}\right\|,
$$


where $C_{11}=1+o(1) \frac{p}{1-\varepsilon}, C_{11}^{\prime}=\frac{C_{9} p}{1-\varepsilon}, C_{12}=\frac{\overline{C_{\varepsilon}}\left\|u_{\lambda}\right\|^{p}+p C_{10}}{1-\varepsilon}$. So we get

$$
\left\|v_{n}\right\|^{p}-C_{13}\left\|v_{n}\right\|^{p-1} \leq C_{12}+o(1)\left\|u_{\lambda}\right\|
$$

where $C_{13}=C_{11}+C_{11}^{\prime}$. It shows that $\left\{v_{n}\right\}$ is bounded in $W_{0}^{1, p}(\Omega)$, going if necessary to a subsequence, one gets that

$$
\left\{\begin{array}{l}
v_{n} \rightarrow v_{0} \text { weakly in } W_{0}^{1, p}(\Omega) \\
v_{n} \rightarrow v_{0} \text { strongly in } L^{\gamma}(\Omega), 1<\gamma<p^{*} \\
v_{n} \rightarrow v_{0} \text { a.e. in } \Omega
\end{array}\right.
$$

as $n \rightarrow \infty$

In addition, by the Sobolev embedding theorem, there exists $M^{\prime}>0$ such that $\left\|u_{\lambda}+v_{n}^{+}\right\|_{p^{*}(s)}^{p^{*}(s)} \leq M^{\prime}$, denote by meas $E$ the measure of $E$. By $\left(A_{1}\right)$, for any $\varepsilon>0$, there exists $C_{14}(\varepsilon)>0$ such that

$$
|f(x, t) t| \leq C_{14}(\varepsilon)+\frac{\varepsilon}{2 M^{\prime}}|t|^{p^{*}(s)},(x, t) \in \bar{\Omega} \times R .
$$

Set $\delta:=\frac{\varepsilon}{2 C_{14}(\varepsilon)}>0$, when $E \subset \Omega$, meas $E<\delta$, we have

$$
\begin{aligned}
\left|\int_{E} f\left(x, u_{\lambda}+v_{n}^{+}\right)\left(u_{\lambda}+v_{n}^{+}\right) d x\right| & \leq \int_{E}\left|f\left(x, u_{\lambda}+v_{n}^{+}\right)\left(u_{\lambda}+v_{n}^{+}\right)\right| d x \\
& \leq \int_{E} C_{14}(\varepsilon) d x+\frac{\varepsilon}{2 M^{\prime}} \int_{E}\left|u_{\lambda}+v_{n}^{+}\right|^{p^{*}(s)} d x \\
& \leq C_{14}(\varepsilon) \text { meas } E+\frac{\varepsilon}{2}<\varepsilon .
\end{aligned}
$$

By Vitali's theorem, we prove that

$$
\int_{\Omega} f\left(x, u_{\lambda}+v_{n}^{+}\right)\left(u_{\lambda}+v_{n}^{+}\right) d x \rightarrow \int_{\Omega} f\left(x, u_{\lambda}+v_{0}^{+}\right)\left(u_{\lambda}+v_{0}^{+}\right) d x \text { as } n \rightarrow \infty .
$$

Hence one has

$$
\begin{aligned}
& \int_{\Omega} f\left(x, u_{\lambda}+v_{n}^{+}\right)\left(u_{\lambda}+v_{n}\right) d x \\
& =\int_{\Omega} f\left(x, u_{\lambda}+v_{n}^{+}\right)\left(u_{\lambda}+v_{n}^{+}\right) d x-\int_{\Omega} f\left(x, u_{\lambda}\right)\left(v_{n}^{-}\right) d x \\
& \rightarrow \int_{\Omega} f\left(x, u_{\lambda}+v_{0}^{+}\right)\left(u_{\lambda}+v_{0}\right) d x \text { as } n \rightarrow \infty .
\end{aligned}
$$

Using the same method, we deduce that

$$
\begin{aligned}
\int_{\Omega} F\left(x, u_{\lambda}+v_{n}^{+}\right) d x & \rightarrow \int_{\Omega} F\left(x, u_{\lambda}+v_{0}^{+}\right) d x, \\
\int_{\Omega} f\left(x, u_{\lambda}+v_{n}^{+}\right) \omega d x & \rightarrow \int_{\Omega} f\left(x, u_{\lambda}+v_{0}^{+}\right) \omega d x,
\end{aligned}
$$

as $n \rightarrow \infty$ for $\omega \in W_{0}^{1, p}(\Omega)$. Hence, similar to the proof of Theorem 1, we have

$$
0=\lim _{n \rightarrow \infty}\left\langle J^{\prime}\left(v_{n}\right), \omega\right\rangle=\left\langle J^{\prime}\left(v_{0}\right), \omega\right\rangle
$$

for $\omega \in W_{0}^{1, p}(\Omega)$, which implies that $J^{\prime}\left(v_{0}\right)=0$. Therefore, $v_{0}$ is a critical point of $J$ in $W_{0}^{1, p}(\Omega)$. From the assumption that $v=0$ is the only critical point of $J$, we know that $v_{0}=0$. Now we want to prove $v_{0} \rightarrow 0$ strongly in $W_{0}^{1, p}(\Omega)$. From (3.10), (3.12) and the Brezis-Leib Lemma (see [3]), we have

$$
J\left(v_{n}\right)=\frac{1}{p}\left\|v_{n}^{-}\right\|^{p}+I\left(u_{\lambda}+v_{n}^{+}\right)-I\left(u_{\lambda}\right)=\frac{1}{p}\left\|v_{n}\right\|^{p}-\frac{\mu}{p^{*}(s)} \int_{\Omega} \frac{\left(v_{n}^{+}\right)^{p^{*}(s)}}{|x|^{s}} d x+o(1) .
$$


Therefore, we get

$$
\left\langle J^{\prime}\left(v_{n}\right), v_{n}\right\rangle=\left\|v_{n}\right\|^{p}-\mu \int_{\Omega} \frac{\left(v_{n}^{+}\right)^{p^{*}(s)}}{|x|^{s}} d x+o(1) \rightarrow 0,
$$

then $\left\|v_{n}\right\|^{p} \rightarrow 0$ as $n \rightarrow \infty$. Otherwise, there exists a subsequence (still denoted by $v_{n}$ ) such that

$$
\lim _{n \rightarrow \infty}\left\|v_{n}\right\|^{p}=k, \quad \lim _{n \rightarrow \infty} \mu \int_{\Omega} \frac{\left(v_{n}^{+}\right)^{p^{*}(s)}}{|x|^{s}} d x=k, k>0 .
$$

By (1.2), we deduce that

$$
\left\|v_{n}\right\|^{p} \geq A_{s}\left(\int_{\Omega} \frac{\left(v_{n}^{+}\right)^{p^{*}(s)}}{|x|^{s}} d x\right)^{\frac{p}{p^{*}(s)}} \quad, \quad \text { for all } n \in N .
$$

Then, $k \geq A_{s}\left(\frac{k}{\mu}\right)^{\frac{p}{p^{*}(s)}}$, that is, $k \geq A_{s}^{\frac{N-s}{p-s}} \mu^{\frac{p-N}{p-s}}$. Thus we get that

$$
\begin{aligned}
c=o(1)+J\left(v_{n}\right) & =\frac{1}{p}\left\|v_{n}\right\|^{p}-\frac{\mu}{p^{*}(s)} \int_{\Omega} \frac{\left(v_{n}^{+}\right)^{p^{*}(s)}}{|x|^{s}} d x+o(1) \\
& =\frac{p-s}{p(N-s)} k+o(1) \\
& \geq \frac{p-s}{p(N-s)} A_{s}^{\frac{N-s}{p-s}} \mu^{\frac{p-N}{p-s}} .
\end{aligned}
$$

This is a contradiction. So $v_{n} \rightarrow 0$ strongly in $W_{0}^{1, p}(\Omega)$ as $n \rightarrow \infty$.

Since $u_{\lambda}>0$ is a solution of problem (1.1), in a way similar to the proof of Theorem 1.1 in [5], we obtain positive constants $R$ and $r_{0}$ such that $B_{2 R}(0) \subset \Omega$ and

$$
0<r_{0} \leq u_{\lambda}(x), \forall x \in B_{2 R}(0) \backslash\{0\} .
$$

In the following, we shall give some estimates for the extremal functions. Let

$$
C_{\varepsilon}:=\left[\varepsilon(N-s)\left(\frac{N-p}{p-1}\right)^{p-1}\right]^{\frac{N-p}{p(p-s)}}, U_{\varepsilon}(x):=\frac{l_{\varepsilon}(x)}{C_{\varepsilon}} .
$$

Define a function $\varphi \in C_{0}^{\infty}(\Omega), 0 \leq \varphi(x) \leq 1$ such that

$$
\varphi(x)=\left\{\begin{array}{l}
1,|x| \leq R \\
0,|x| \geq 2 R
\end{array}\right.
$$

where $B_{2 R}(0) \subset \Omega$. Set

$$
u_{\varepsilon}(x):=\varphi(x) U_{\varepsilon}(x), \quad v_{\varepsilon}(x):=\frac{u_{\varepsilon}(x)}{\left(\int_{\Omega} \frac{\left|u_{\varepsilon}\right| p^{*}(s)}{|x|^{s}} d x\right)^{\frac{1}{p^{*}(s)}}},
$$

so that $\int_{\Omega} \frac{\left|v_{\varepsilon}\right|^{p^{*}(s)}}{|x|^{s}} d x=1$. Then, by using the argument as $[\mathbf{9}]$, we can get the following results:

$$
A_{s}+C_{15} \varepsilon^{\frac{N-p}{p-s}} \leq\left\|v_{\varepsilon}\right\|^{p} \leq A_{s}+C_{16} \varepsilon^{\frac{N-p}{p-s}}
$$

and

$$
C_{17}\left(\varepsilon^{\frac{(p-s)(p-1)}{p}}\right) \leq \int_{\Omega} \frac{\left|v_{\varepsilon}\right|^{p}}{|x|^{s}} d x \leq C_{18}\left(\varepsilon^{\frac{(p-s)(p-1)}{p}}\right), p>\frac{N-s}{N-p}(p-1) .
$$


Lemma 4. Suppose that $N \geq 3,0<\mu<\infty$ and $\max \left\{0, \frac{p^{2}-N}{p-1}\right\}<s<p$. Assume $\left(A_{1}\right)-\left(A_{3}\right)$ and $f(x, 0) \equiv 0$ hold. Then there exists $v_{*} \in W_{0}^{1, p}(\Omega)$, $v_{*} \not \equiv 0$, such that

$$
\sup _{t \geq 0} J\left(t v_{*}\right)<\frac{p-s}{p(N-s)} A_{s}^{\frac{N-s}{p-s}} \mu^{\frac{p-N}{p-s}} .
$$

Proof. By (3.2), $\left(A_{2}\right)$ and an elementary inequality (the proof of this inequality is elementary but not straightforward. We postpone it to Appendix A.)

$(a+b)^{\gamma} \geq a^{\gamma}+b^{\gamma}+C a^{\gamma-t} b^{t}, \gamma \geq 2,1 \leq t \leq \gamma-1, a, b>0$, where $C$ is a positive constant, we have

$$
g(x, l) \geq \mu \frac{l^{p^{*}(s)-1}}{|x|^{s}}+C \mu \frac{l^{p-1} u_{\lambda}^{p^{*}(s)-p}}{|x|^{s}},
$$

where $\left(A_{3}\right)$ implies $p^{*}(s)-1 \geq 2,1 \leq p-1 \leq\left(p^{*}(s)-1\right)-1$. Therefore, we have

$$
G\left(x, t v_{\varepsilon}\right) \geq \mu \frac{t^{p^{*}(s)}}{p^{*}(s)} \frac{v_{\varepsilon}^{p^{*}(s)}}{|x|^{s}}+\frac{C \mu t^{p}}{p} \frac{v_{\varepsilon}^{p} u_{\lambda}^{p^{*}(s)-p}}{|x|^{s}} .
$$

Note that $s>\frac{p^{2}-N}{p-1}$ implies $p>\frac{N-s}{N-p}(p-1)$, then (3.15) holds. Therefore, from (3.13)-(3.15), we deduce that

$$
\begin{aligned}
J\left(t v_{\varepsilon}\right) & =\frac{t^{p}}{p}\left\|v_{\varepsilon}\right\|^{p}-\int_{\Omega} G\left(x, t v_{\varepsilon}\right) d x \\
& \leq \frac{t^{p}}{p}\left\|v_{\varepsilon}\right\|^{p}-\mu \frac{t^{p^{*}(s)}}{p^{*}(s)}-C_{19} t^{p} \int_{\Omega} \frac{v_{\varepsilon}^{p}}{|x|^{s}} d x \\
& \leq \frac{t^{p}}{p}\left\|v_{\varepsilon}\right\|^{p}-\mu \frac{t^{p^{*}(s)}}{p^{*}(s)}-C_{20} t^{p} \varepsilon^{\frac{(p-s)(p-1)}{p}} \\
& \leq \frac{A_{s}}{p} t^{p}+C_{21} t^{p} \varepsilon^{\frac{N-p}{p-s}}-\mu \frac{t^{p^{*}(s)}}{p^{*}(s)}-C_{20} t^{p} \varepsilon^{\frac{(p-s)(p-1)}{p}},
\end{aligned}
$$

where $C_{19}=\frac{C \mu r_{0}^{p^{*}(s)-p}}{p}, C_{20}=C_{17} C_{19}$ and $C_{21}=\frac{C_{16}}{p}$. Let

$$
Q(t):=\frac{A_{s}}{p} t^{p}+C_{21} t^{p} \varepsilon^{\frac{N-p}{p-s}}-\mu \frac{t^{p^{*}(s)}}{p^{*}(s)}-C_{20} t^{p} \varepsilon^{\frac{(p-s)(p-1)}{p}} .
$$

It is clear that the equation

$$
0=Q^{\prime}(t)=A_{s} t^{p-1}+p C_{21} t^{p-1} \varepsilon^{\frac{N-p}{p-s}}-\mu t^{p^{*}(s)-1}-p C_{20} t^{p-1} \varepsilon^{\frac{(p-s)(p-1)}{p}}
$$

has only one positive root

$$
t_{\varepsilon}:=\left(\frac{A_{s}+p C_{21} \varepsilon^{\frac{N-p}{p-s}}-p C_{20} \varepsilon^{\frac{(p-s)(p-1)}{p}}}{\mu}\right)^{\frac{1}{p^{*}(s)-p}} .
$$


We have

$$
\begin{aligned}
Q\left(t_{\varepsilon}\right) & =\frac{1}{p}\left(A_{s}+p C_{21} \varepsilon^{\frac{N-p}{p-s}}-p C_{20} \varepsilon^{\frac{(p-s)(p-1)}{p}}\right) t_{\varepsilon}^{p}-\mu \frac{t_{\varepsilon}^{p^{*}(s)}}{p^{*}(s)} \\
& =\mu\left(\frac{1}{p}-\frac{1}{p^{*}(s)}\right)\left(\frac{A_{s}+p C_{21} \varepsilon^{\frac{N-p}{p-s}}-p C_{20} \varepsilon^{\frac{(p-s)(p-1)}{p}}}{\mu}\right)^{\frac{p^{*}(s)}{p^{*}(s)-p}} \\
& =\frac{p-s}{p(N-s)} \mu^{\frac{p-N}{p-s}}\left(A_{s}+p C_{21} \varepsilon^{\frac{N-p}{p-s}}-p C_{20} \varepsilon^{\frac{(p-s)(p-1)}{p}}\right)^{\frac{N-s}{p-s}} \\
& <\frac{p-s}{p(N-s)} \mu^{\frac{p-N}{p-s}} A_{s}^{\frac{N-s}{p-s}},
\end{aligned}
$$

for $\varepsilon>0$ sufficiently small due to the fact that

$$
\frac{N-p}{p-s}>\frac{(p-s)(p-1)}{p}, \text { for } s>\frac{p^{2}-N}{p-1} .
$$

Noting that $Q(0)=0$ and $\lim _{t \rightarrow+\infty} Q(t)=-\infty$, we have

$$
\sup _{t \geq 0} Q(t)=Q\left(t_{\varepsilon}\right)<\frac{p-s}{p(N-s)} \mu^{\frac{p-N}{p-s}} A_{s}^{\frac{N-s}{p-s}},
$$

for $\varepsilon>0$ sufficiently small. Hence we obtain

$$
\sup _{t \geq 0} J\left(t v_{\varepsilon}\right) \leq \sup _{t \geq 0} Q(t)<\frac{p-s}{p(N-s)} \mu^{\frac{p-N}{p-s}} A_{s}^{\frac{N-s}{p-s}},
$$

for $\varepsilon>0$ sufficiently small, which complete the proof by letting $v_{*}=v_{\varepsilon}$ for $\varepsilon>0$ sufficiently small.

Proof of Theorem 2. By contradiction. Assume that $v=0$ is the only critical point of $J$ in $W_{0}^{1, p}(\Omega)$. From Lemma 1, there exists $\alpha>0$ such that $J(v)>\alpha$ for all $v \in \partial B_{\rho}=\left\{v \in W_{0}^{1, p}(\Omega),\|v\|=\rho\right\}$, where $\rho>0$ small enough. By Lemma 4 there exists $v_{*} \in W_{0}^{1, p}(\Omega), v_{*} \not \equiv 0$, such that

$$
\sup _{t \geq 0} J\left(t v_{*}\right)<\frac{p-s}{p(N-s)} A_{s}^{\frac{N-s}{p-s}} \mu^{\frac{p-N}{p-s}} .
$$

From (3.8), we easily note that $\lim _{t \rightarrow \infty} J\left(t v_{*}\right) \rightarrow-\infty$. Hence we can choose $t_{0}>0$ such that $\left\|t_{0} v_{*}\right\|>\rho$ and $J\left(t_{0} v_{*}\right)<0$. Applying the Mountain Pass Lemma (see [15] or [14]), there is a sequence $\left\{v_{n}\right\} \subset W_{0}^{1, p}(\Omega)$ satisfying

$$
J\left(v_{n}\right) \rightarrow c \geq \alpha \text { and } J^{\prime}\left(v_{n}\right) \rightarrow 0,
$$

where

$$
c=\inf _{h \in \Gamma} \max _{t \in[0,1]} J(h(t))
$$

and

$$
\Gamma=\left\{h \in C([0,1], X) \mid h(0)=0, h(1)=t_{0} v_{*}\right\}
$$

Note that

$$
\begin{aligned}
& 0<\alpha \leq c=\inf _{h \in \Gamma} \max _{t \in[0,1]} J(h(t)) \\
& \leq \max _{t \in[0,1]} J\left(t t_{0} v_{*}\right) \leq \sup _{t \geq 0} J\left(t v_{*}\right)<\frac{p-s}{p(N-s)} A_{s}^{\frac{N-s}{p-s}} \mu^{\frac{p-N}{p-s}} .
\end{aligned}
$$


Together with Lemma 3 , we know that $v_{n} \rightarrow 0$ strongly in $W_{0}^{1, p}(\Omega)$ as $n \rightarrow \infty$. Hence one has $0=J(0)=\lim _{n \rightarrow \infty} J\left(v_{n}\right)=c \geq \alpha>0$, this is a contradiction. So Theorem 2 holds.

\section{Appendix A}

Here we give the proof of the elementary inequality in Lemma 4, that is,

$$
(a+b)^{\gamma} \geq a^{\gamma}+b^{\gamma}+C a^{\gamma-t} b^{t}, \gamma \geq 2,1 \leq t \leq \gamma-1, a, b>0,
$$

where $C$ is a positive constant.

Proof. Indeed, by scaling it suffices to show that

$$
(1+x)^{\gamma} \geq 1+x^{\gamma}+C x^{t}, 0<x<\infty .
$$

Let $\gamma=k+\theta, t=m+\eta$, where $k \geq 2,1 \leq m \leq k-1$ are integral numbers and $0 \leq \eta \leq \theta<1$ are real numbers. It is obvious that

$$
\begin{aligned}
(1+x)^{\gamma}=(1+x)^{k+\theta} & =(1+x)^{k}(1+x)^{\theta} \\
& \geq\left(1+x^{k}+C x^{m}\right)(1+x)^{\theta} \\
& \geq 1+x^{k+\theta}+C x^{m}(1+x)^{\theta} \\
& \geq 1+x^{k+\theta}+C x^{m} x^{\eta}=1+x^{\gamma}+C x^{t} .
\end{aligned}
$$

Therefore, this inequality holds.

\section{References}

[1] A. Ambrosetti, H. Brezis, G. Cerami, Combined effects of concave and convex nonlinearities in some elliptic problems, J. Funct. Anal., 122 (2) (1994), 519-543.

[2] H. Brezis, L. Nirenberg, Positive solutions of nonlinear elliptic equations involving critical Sobolev exponents, Comm. Pure Appl. Math., 36 (4) (1983), 437-477.

[3] H. Brezis, E. Lieb, A relation between pointwise convergence of functions and convergence of functionals, Proc. Amer. Math. Soc., 88 (3) (1983), 486-490.

[4] L. Caffarelli, R. Kohn and L. Nirenberg, First Order Interpolation Inequality with Weights, Compositio Math., 53 (1984), 259-275.

[5] J.Q. Chen, Multiple positive solutions for a class of nonlinear elliptic equations, J. Math. Anal. Appl., 295 (2) (2004), 341-354.

[6] Y.B. Deng, L.Y. Jin, Multiple positive solutions for a quasilinear nonhomogeneous Neumann problems with critical Hardy exponents, Nonlinear Anal., 67 (12) (2007), 3261-3275.

[7] H. Egnell, Positive solutions of semilinear equations in cones, Trans. Amer. Math. Soc., 11 (1992), 191-201.

[8] J.V. Goncalves, C.O. Alves, Existence of positive solutions for m-Laplacian equations in $R^{N}$ involving critical Sobolev exponents, Nonlinear Anal., 32 (1) (1998), 53-70.

[9] N. Ghoussoub, C. Yuan, Multiple solutions for quasi-linear PDEs involving the critical Sobolev and Hardy exponents, Trans. Amer. Math. Soc., 352 (12) (2000), 5703-5743.

[10] N. Ghoussoub, X.S. Kang, Hardy-Sobolev critical elliptic equations with boundary singularities, Ann. I. H. Poincaré-AN, 21 (2004), 767-793.

[11] E. Jannelli, The role played by space dimension in elliptic critical problems, J. Differential Equations, 156 (2) (1999), 407-426.

[12] Elves A.B. Silva, Joao C.N. Padua, Sergio H.M. Soares, Positive solutions of critical semilinear problems involving a sublinear term at the origin, Cadernos De Mathematica., 5 (2004), 245262.

[13] J. Mawhin, M. Willem, Critical Point Theory and Hamiltonian Systems, Appl. Math. Sci., vol. 74, Springer, New York, 1989.

[14] Michael Struwe, Variational methods :applications to nonlinear partial differential equations and Hamiltonian systems, Berlin : Springer, 1996. 
[15] P.H. Rabinowitz, Minimax Methods in Critical Point Theory with Applications to Differential Equations, in: CBMS Reg. Conf. Series. Math., vol. 65, Amer. Math. Soc., Providence, RI, 1986.

School of Mathematical Sciences and LPMC, Nankai University, Tianjin 300071, P. R. CHINA

E-mail address: guanweic@163.com

School of Mathematical Sciences and LPMC, Nankai University, Tianjin 300071, P. R. CHINA

E-mail address: shiwangm@163.com 